Mini Review

\title{
Recent Advances and Future Prospects of Aptamer-based Biosensors in Food Safety Analysis
}

\author{
Yue Wang ${ }^{l}$, Hongguo Zhai ${ }^{1}$,Jiaqi Yin ${ }^{l}$, Qi Guo ${ }^{1}$, Yuhao Zhang ${ }^{l}$, Xia Sun ${ }^{1}$, Yemin Guo , \\ Qingqing Yang ${ }^{l}$, Falan Li, Yanyan Zhang ${ }^{1,2,3, *}$ \\ ${ }^{1}$ School of Agricultural Engineering and Food Science, Shandong University of Technology, No.12 \\ Zhangzhou Road, Zibo 255049, Shandong Province, China. \\ ${ }^{2}$ Shandong Xicheng Agricultural Machinery Technology Co., Ltd., Shandong Province, China. \\ ${ }^{3}$ Shandong Sider Agricultural Equipment Co., LTD., Shandong Province, China. \\ *E-mail: zyyan1104@163.com
}

Received: 22 September 2021 / Accepted: 27 October 2021 / Published: 6 December 2021

The problem of environmental pollution and food security have brought global attention to food safety issues. Pollutant detection is one of the significant missions in the background of serious global environmental problems. Heavy metal ions, pesticides, biotoxins, and antibiotics on sites are in high demand in environmental analysis. As a simple, accurate, inexpensive, and selective detection method, aptamer-based biological sensors are very suitable for environmental detection and food safety analysis. This review principally summarizes the recent advances in the use of aptamer-based fluorescence sensors for food safety detection. This includes the detection of divalent metal ions, trivalent metal ions, pesticide leftover, biotoxins, and antibiotics.

Keywords: Biological aptamer sensors, Food safety, Heavy metals, Pesticide residues, Biotoxin, Antibiotics

\section{$\underline{\text { FULL TEXT }}$}

(C) 2022 The Authors. Published by ESG (www.electrochemsci.org). This article is an open access article distributed under the terms and conditions of the Creative Commons Attribution license (http://creativecommons.org/licenses/by/4.0/). 